\title{
Lumen
}

Selected Proceedings from the Canadian Society for Eighteenth-Century Studies

\section{Le théâtre dans le théâtre ou le spectacle de la salle à travers la correspondance de Mme de Graffigny}

\section{Charlotte Simonin}

Volume 22, 2003

URI : https://id.erudit.org/iderudit/1012261ar

DOI : https://doi.org/10.7202/1012261ar

Aller au sommaire du numéro

Éditeur(s)

Canadian Society for Eighteenth-Century Studies / Société canadienne d'étude du dix-huitième siècle

ISSN

1209-3696 (imprimé)

1927-8284 (numérique)

Découvrir la revue

Citer cet article

Simonin, C. (2003). Le théâtre dans le théâtre ou le spectacle de la salle à travers la correspondance de Mme de Graffigny. Lumen, 22, 103-116.

https://doi.org/10.7202/1012261ar

Copyright (c) Canadian Society for Eighteenth-Century Studies / Sociéte canadienne d'étude du dix-huitième siècle, 2003
Ce document est protégé par la loi sur le droit d'auteur. L'utilisation des services d'Érudit (y compris la reproduction) est assujettie à sa politique d'utilisation que vous pouvez consulter en ligne.

https://apropos.erudit.org/fr/usagers/politique-dutilisation/ 


\section{Le théâtre dans le théâtre ou le spectacle de la salle à travers la correspondance de Mme de Graffigny}

Surtout connue aujourd'hui pour ses Lettres d'une Péruvienne (1747), Mme de Graffigny (1695-1758) éprouva pour le théâtre une passion évidente, et l'un des plus grands, sinon le plus grand bonheur que lui donna son nouveau statut de Parisienne à partir de février 1739 fut de pouvoir assister à des représentations; de simple lectrice, elle devint aussi spectatrice, et spectatrice qui avait, luxe suprême, l'embarras du choix entre les Français, les Italiens, les Foires et l'Opéra. Vivre les succès et les échecs de l'intérieur, voir de ses yeux et entendre de ses oreilles les grands acteurs et actrices du moment, assister aux premières des pièces dont tout le monde parlera, lui procurent une excitation et une joie toujours renouvelées, que la Lorraine exilée fait partager à son fidèle correspondant François Devaux, surnommé Panpan.

Or, avec la plume acérée que l'on lui connaît, l'épistolière ne manque pas d'observer que le spectacle se déroule tout autant, sinon plus parfois, dans la salle que sur la scène. Montesquieu avait déjà moqué ce phénomène dans la célèbre lettre XXVIII de ses Lettres persanes, où Rica s'étonne:

Je vis hier une chose assez singulière, quoiqu'elle se passe tous les jours à Paris. Tout le peuple s'assemble sur la fin de l'après-dînée et va jouer une espèce de scène que j'ai entendu appeler comédie. Le grand mouvement est sur une estrade qu'on nomme le théâtre. Aux deux côtés, on voit, dans de petits réduits qu'on nomme loges, des hommes et des femmes qui jouent ensemble des scènes muettes, à peu près comme celles qui sont en usage dans notre Perse.

[...] Il y a en bas une troupe de gens debout, qui se moquent de ceux qui sont en haut sur le théâtre, et ces derniers rient à leur tour de ceux qui sont en bas ${ }^{1}$.

1 Montesquieu, Lettres persanes, préface, commentaires et notes de Georges Gusdorf, Paris, Librairie générale française, coll. «Le livre de poche», 1984, p. 58. 
L'on citerait encore les Liaisons dangereuses qui peignent elles aussi le spectacle de la salle: Danceny et Cécile se retrouvent à l'Opéra pendant que Mme de Merteuil lorgne (lettre XII); c'est devant l'Opéra que la voiture de Mme de Tourvel croise celle de Valmont accompagné d'Émilie (lettres CXXXV, CXXXVII et CXXXVIII); c'est enfin à la Comédie-Italienne que la marquise subit l'ultime disgrâce lorsque le public la hue (lettre CLXXIII). Puisque les deux exemples précédents proviennent de deux romans, $l^{\prime}$ on pourrait croire que la fiction exagère le trait, caricature la réalité. Mais les lettres de Mme de Graffigny, sans nulle exagération romanesque, montrent que les comportements dans les salles de spectacles sont en effet spectaculaires, théâtralisés ${ }^{2}$. Nous rapporterons ici les anecdotes les plus saillantes narrées par «La Grosse» concernant ce véritable théâtre dans le théâtre et nous tenterons de saisir les codes de comportement (dits et non dits) régissant ce public composite réparti entre scène (espace de montre chéri des petits-maîtres), amphithéâtre, loges (grillées, elles dissimulent les amants en rendez-vous galants), parterre et paradis ${ }^{3}$.

\section{Le spectacle du parterre}

Nous nous intéresserons bien sûr particulièrement au parterre ${ }^{4}$, aux rites bruyants par lesquels il marque son plaisir ou sa colère: mouvements divers (ondes...), danses, acclamations, huées ou larmes. Le parterre, tel un enfant incontrôlable, offre un miroir grossissant, déformé des attitudes policées, adultes des autres spectateurs, mais un miroir néan-

2 Toute la Correspondance de Mme de Graffigny est publiée par la Voltaire Foundation, Oxford: Tome I (1716-17 juin 1739), préparé par E. Showalter, 1985, 592 p.; Tome II (19 juin 1739-24 septembre 1740), préparé par J. A. Dainard et E. Showalter, 1989, 505 p.; Tome III ( $1^{\mathrm{er}}$ octobre 1740-27 novembre 1742) préparé par N. R. Johnson, 1992, 498 p.; Tome IV (30 novembre 1742-2 janvier 1744), préparé par J. A. Dainard, M.-P. Ducretet et E. Showalter, 1996, 592 p.; Tome V (3 janvier 1744-21 octobre 1744) préparé par J. Curtis, 1997, 578 p.; Tome VI (23 octobre 1744-10 septembre 1745) préparé par P. Bouillaguet, J. Curtis et J. A. Dainard, 2000, 638 p. Je cite le volume en chiffres romains, et la page en chiffres arabes, et respecte la graphie de Mme de Graffigny.

3 Voir aussi sur ce sujet l'excellent ouvrage de Henri Lagrave, le Théatre et le public à Paris de 1715 à 1750, Paris, Klincksieck, 1972.

4 Le mot intervient aussi sous la plume de Mme de Graffigny dans l'acception de jardin (lettres du 10 septembre et du 25 octobre 1749, quand elle décrit la petite maison qu'elle loue près de Choisy). 
moins. Son comportement tribal met véritablement en scène la réception de la pièce et il interdit ou garantit le succès.

Aujourd'hui, le public qui assiste à une pièce de théâtre s'impose un silence religieux; qui laisse échapper le moindre chuchotement ou la moindre quinte de toux se fait assassiner de regards furieux. Au XVIII siècle, il en va bien différemment; le public, et surtout le parterre, rivalise par son niveau sonore avec le spectacle qui se déroule sur scène, au point que ce dernier en devient parfois inaudible. En effet, le parterre revendique sa présence par divers cris et bruits. Les applaudissements saluent l'entrée en scène des acteurs vedettes du moment: "Arrive la Comtesse qui tourne le dos pour heurter à la porte de Dandin. Personne ne devine qui c'est: elle se retourne, c'est la divine Quinaud! Le pauvre petit partere fait un bruit, Dieu sait, et moi je saute de joye» (II, 81); «Il [Deshayes] est recu du partere comme l'etoit Dufrene. Des qu'il paroit, les mains vont» (III, 431). Ou tel ou tel morceau de bravoure: «Il a falu que La Nouè repete quatre vers qui finit l'acte par un monologue que des aplaudissements avoient empeché d'entendre» $(\mathrm{V}, 234)$. Ces frappements des mains se doublent parfois de tapements des pieds - Mme de Graffigny note plaisamment les risques pour le sol de bois: "Aussi casse-t-on les planches pour l'aplaudir [Mlle Abeille]» (III, 425); ou de cris: «La Gaussin fut reçue avec de grand applaudissements, mais elle ne le fut pas une fois dans le cours du role, et Clairon le fut des pieds, des mains, de la voix - enfin le grand train» (IV, 482). Le parterre rit aussi avec fracas, mais le reste de la salle ne partage manifestement pas cet humour, puisque Mme de Graffigny indique bien que seul le parterre rit: «- Leurs gens? Ces gens ont-ils des gens? - Oui, monsieur, leurs gens sont des sergents. Et le partere de rire $(\mathrm{V}, 232)^{5}$ ». Qu'il manifeste son approbation ou son mécontentement, le parterre reste bruyant. Les murmures et les brouhahas précèdent les huées: «Cet acte est detestable pour les choses et pour le ton; on n'a cependant pas hué, mais des murmures confus faisoient tout craindre» $(\mathrm{V}, 235)$. Sans doute trop novatrice, la Dispute de Marivaux chute avec fracas dès la première représentation, le 19 octobre 1744: «Un monsieur duc qui en venoit a dit que, depuis qu'il se connoit et qu'il va a la commedie, et il y va souvent, il n'a jamais vu une huée si cruelle. Il y avoit des siflet, ce qui n'est plus $\mathrm{d}^{\prime}$ usage» $(\mathrm{V}, 526)$. Le parterre pousse aussi la chansonnette à l'occasion:

5 A propos d'une adaptation de Pamela, elle parle d'un vers qui fait «rire et clabauder le partere» (IV, 507). 
Entre les deux piece on joua par hazard «Viens dans ma celule»; voila le partere a chanter et a sauter sur les talons, ne pouvant faire mieux, de façon que c'etoit un bruit a en etre sourd, outre la poussiere, qui etoit a etouffer. Il firent reccommencer dix fois l'air (II, 286).

Inépuisable, le parterre peut hurler des heures durant:

Le partere crie sans fin et sans relache: «Mille coups de baton! Mille coups de baton!» tant que l'exemp se retire comme Sosie. Je n'en sais pas plus mais a dix heure ce train-la duroit encore, et tout le peuple assemblé dans la ruè d'ou l'on entendoit les cris du parterre. Nini, qui y etoit, en est sortie a dix heures voyant que l'on n'avancoit ny ne reculoit. Quand je saurai le reste je te le dirai (V, 283).

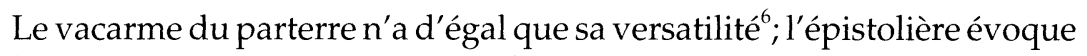
la réception contrastée de Fernand Cortez de Piron:

Elle [la pièce] a eté huée, bafouée, pendant le premier, second et troizieme actes, qui sont chaqu'un de la longueur d'une piece. Il y avoit apparance qu'on ne la laisseroit pas finir. [...] Ce morceau a reconcilié l'auteur avec le public, on a beaucoup a[pplaudi]. [...] Cependant sa cabale l'a emporté: on l'a demandée pour samedi ${ }^{7}(\mathrm{~V}, 15)$.

Le parterre évacue aussi son trop-plein d'énergie par divers mouvements, qu'il s'agisse d'ondes (les spectateurs imitent le mouvement de la mer en ondulant comme des vagues) -

Ils ont fait des ondes tant qu'ils ont pu. Il y avoit de la place de reste; cependant ils avoient resolu d'aplatir ceux qui touchoient l'orquestre, mais ils n'ont pas reussit car ils etoient aussi fort - et les ondes d'aller. Je l'avoue, le partere me diverti de bon cœur (I, 369) -

\section{ou carrément de danse -}

Puisque les folies du partere $t^{\prime}$ amuse, en voici une de dimanche. Apres des flus et des reflus sans nombres, ils parvinrent a menager une petite place au beau milieu et se mirent cinq ou six a danser en rond, marchant sur les malheureux

6 Lors d'une représentation d'Inès de Castro, Mme de Graffigny note: «le partere a ri a deux ou trois endroit, surtout a l'arrivée des enfants, et a pleuré un moment apres comme un sot», mais ajoute avec franchise «J'en ai fait de même» (II, 79).

7 Mme de Graffigny a vu la pièce le mercredi 8 janvier 1744 . 
pieds qui se trouvoient les plus pres, et forcerent l'orquestre de leur jouer des airs qu'ils vouloient (IV, 26).

Si Mme de Graffigny s'amuse parfois du parterre, elle est souvent, en véritable amatrice de théâtre qu'elle est, excédée par ce parasitage constant de la pièce:

Mais si j'avois eu des foudres, je les aurois lancé sur le partere. Imagine donc qu'il a toujours crié, siflé, batu des mains a chaque mot que dit Dubreuil, qui jouoit Corasmin, sans egard a aucune belle situation, qui par là ont toutes eté gatée car il faloit que Grandval s'aretat chaque fois avant de parler (IV, 220).

Ainsi, elle use fréquemment d'expressions péjoratives pour le qualifier: maudit parterre, chien de parterre: «La Clairon, qui fait le recit de la fin, le crie, le hurle. Le chien de partere l'applaudit et les gens de bon sens sont toujours pres a lui demander ce qu'elle a a crier puisque tout va bien. Cela est horrible» $(\mathrm{V}, 88)$. Qu'elle soit amusée ou énervée, elle a en tout cas tout à fait conscience que le parterre constitue un spectacle à part entière, et elle emploie le terme: «Cependant rien ne manquoit de ce qui pouvoit $\mathrm{m}^{\prime}$ amuser. On fut lontems a comenser. Le partere bati cinq ou six fois des mains. Il sembloit qu'il vouloit me donner son spectacle aussi, car il n'a jamais été si impertinent et j'avoue qu'il m'amusa» (I, 322).

À la fin d'une pièce, le parterre apostrophe parfois les acteurs en criant "Annonce» (par exemple, II, 39 ou III, 258), ce qui signifie que la pièce jouée déplaît et qu'il faut en annoncer une nouvelle pour le lendemain. Le parterre n'hésite pas à critiquer le jeu des acteurs:

Un acteur nouveau debutoit dans Radamiste sous la protection de la charmante Gossin. [...] A la fin de la piece, une voix crie dans le parterre: «Voila un mauvais acteur!» Je ne sais encore pourquoi ny comment, l'exemp a eté arreter cette voix, et l'a conduit au Châtelet. Grande rumeur. Les acteurs paroissent, qui etoit Harmand et le nouvel acteur, pour commencer Amour pour amour. On les huè, on les chasse avec fureur et on redemande 1'homme arreté. [...] Ce chien de public en fureur crie qu'il ne permettront pas de jouer pendant une eternitté s'il le faut, tant qu'ils n'auront pas l'homme. Il crie aux acteurs qu'ils ne s'avisent pas de se deshabiller ny de jouer, qu'ils coucheront la. Cela dure une heure, toujours en conversation avec l'humble Harmand, qui etoit prosterné. L'emeute ogmente; le parterre demande a voir l'exemp [...] $(\mathrm{V}, 283)$.

La pièce est interrompue tant que l'on n'a pas obtempéré.

Le parterre apostrophe aussi parfois les spectateurs. Le refrain célèbre «Place au...» connaît nombre de variations. Le plus souvent, il s'agit de «Place au théâtre», le théâtre étant généralement si encombré de petitsmaîtres paradant qu'il ne reste guère de place pour les acteurs tentant 
de s'avancer, ou de «Place aux dames ${ }^{8}$ » pour s'écarter sur le passage des beautés du jour. Le parterre demande aussi fréquemment «Ouvrez les loges». En effet, les spectateurs du parterre souffrent de la promiscuité d'un espace confiné et souvent bourré plus que de raison; incommodés par la chaleur, ils interpellent les spectateurs des loges pour qu'ils ouvrent les fenêtres à l'arrière de celles-ci. Certes, ces aérations paraissent plus virtuelles que réelles, vu les rues encaissées et sales sur lesquelles donnent les théâtres, mais cette exigence d'un air plus frais et plus pur (?) est surtout l'occasion pour le parterre d'exercer son pouvoir sur les spectateurs des loges, renversant ainsi la hiérarchie sociale qui existe au dehors du théâtre. Comme le note Mme de Graffigny, ces canicules ne surviennent pas seulement par temps chaud, ici en avril «Avec cela, il se mit en tete, ce maudit partere, de faire ouvrir les loges. Il a falu qu'une fois la Gossin se tourna pour parler a l'exempt apres avoir attendu un gros quart d'heure» (IV, 220) -, mais aussi en plein hiver, en décembre 1742 par exemple: «et ne sachant plus que faire, ils crierent pendant un quart d'heure d'ouvrir les loges comme au mois d'aoust, et on y gelait. Il est trop fou aussi» (IV, 26).

Un jour où elle rapporte d'abord à Panpan tout ce qui s'est passé en attendant la pièce, à l'entracte et entre les deux pièces, avant de raconter la pièce, elle termine par ce concetto: «Voila l'histoire du partere; voici celle de la comedie» (II, 286). La symétrie de la formulation (adverbe déictique, répétition) montre qu'elle a bien conscience qu'il y a (au moins) deux spectacles dans la salle.

\section{Le spectacle des loges}

Les spectatrices des premières loges (aristocrates surtout) et surtout celles assises sur le devant des dites loges se doivent (c'est-à-dire doivent au public qui les admire) d'être parées de leurs plus belles robes et de leurs plus étincelants bijoux. Elles ont pour fonction d'orner la salle. Comme leur présence attire les spectateurs masculins, elles bénéficient d'abonnements à tarifs préférentiels. Ce n'est plus guère le cas aujourd'hui au théâtre, mais on constate toujours le même genre de flirt dans les boîtes de nuit où l'entrée gratuite ou à mi-prix pour les femmes le vendredi ou le samedi soir est pratiquée. L'anecdote suivante montre que la fonction décorative exigée des femmes s'étend par-delà leurs atours à leur apparence même:

8 «Les ondes, les "Place aux dames!" ne finirent pas» (II, 286). 
À propos, tu ne me disputeras plus de n'avoir pas vu le partere plus fou que tu ne l'as vu. Aux François, s'entant, il a fait sortir une femme d'une loge parce qu'il y en avoit une jolie derriere elle, en criant: «Place a la belle!» Cela a duré un tems infini. Les acteurs on comensé, ils se sont tuts; mais a l'autre entreacte, voiant qu'elle n'avoit pas changé de place, ils ont recommensé. Les acteurs sont venus - cela n'a rien fait. Ils n'ont point cessé que cette pauvre dame ne fut sortie, comme s'il n'etoit pas permis d'etre laide. Tout le monde a dit que pareille chose n'etoit jamais arrivée. C'etoit aux secondes loges ${ }^{9}$, comme tu crois (I, 369).

Le parterre réclame comme un enfant gâté, sans se soucier des conséquences, cruelles pour la laide, et embarrassantes pour la belle, qui s'était certainement assise derrière pour des raisons de préséance. C'est aussi Mme de Graffigny qui, victime de la méchanceté de Mme du Châtelet, se voit refuser une place de devant de la loge lors d'une répétition de Dardanus à l'opéra: «La pauvre Grosse, sans repliquer, se mit derriere comme une femme de chambre, avec la douleur dans l'ame» (II, 233). La souffrance se redouble parce que le public a assisté au spectacle de son humiliation: «J'ai vu mille gens de ceux qui vienent ici. Ah, que cela m'a eté sensible! [...] Il y en avoit mille, il est vray, dans presque toute les loges. [...] Me voilà affichée» (II, 243).

Ainsi l'épistolière, un jour où elle n'est pas suffisamment bien vêtue (ce qui montre bien qu'on y va pour se faire regarder tout autant que pour voir), s'interdit-elle sponte sua de figurer aux premières loges et se retrouve avec des voisines un peu trop galantes qui appâtent le chaland:

Nous avons eté confondues a l'opera. Nous ne nous sommes pas trouvée assés bien mise pour aller dans les premieres des premieres. Finement, nous avons pris celle de l'angle, qui sont celle que les directeurs donnent. Moyennant quoi,

9 Le parterre, si irrévérencieux soit-il, connaît les limites. Commec'est une seconde loge, les deux spectatrices, la laide et la belle, sont nécessairement des bourgeoises, l'on peut donc railler sans craindre de représailles. L'incise de Mme de Graffigny, "comme tu crois", montre que le parterre n'aurait certainement pas osé le même comportement avec des aristocrates des premières loges. En effet, les secondes loges n'accueillent qu'exceptionnellement des aristocrates, par exemple à la Foire St-Germain, lors du très grand succès de la parodie de Favart, Acajou: «Je ne sais si tu sera contant d'Acajou, mais il n'y a point de proportion entre le plaisir qu'il m'a fait et la prodigieuse quantité de carosses qui innonde le faubourg Saint-Germain. Hier Md. de Maurepas n'y a pas trouvé place. Les loges sont retenuè en total depuis plus de douze jours jusqu'a demain. Les secondes loges, qui n'ont jamais reçu que de tres petites bourgeoises dans leur enclos, sont a present tres dignes des duchesses. $C^{\prime}$ est une fureur qui dure depuis le premier jour» $(\mathrm{V}, 165)$. 
nous avons eu des compagnes. Ah, quelles compagnes! Des filles! Enfin, nous etions confondues, et pour surcroit Mr de Bresé, que je n'avois pas encore rencontré, m'a vu et m'est venu dire qu'il ne pouvoit croire que ce fut moi, eu egard a la compagnie (II, 201).

Nombre d'intrigues se nouent en effet dans la salle ${ }^{10}$, après des œillades ou des sourires échangés, et grâce à cet indispensable auxiliaire de la séduction qu'est la lorgnette ${ }^{11}$. Et si Manon Lescaut aime tant l'opéra ${ }^{12}$, c'est sans doute plus parce qu'elle y vient recruter de futurs protecteurs que par mélomanie, quoi qu'en pense le crédule Des Grieux.

\section{Le spectacle du gratis}

Le public réellement populaire, pour des raisons à la fois pratiques (manque de temps) et financières (les places sont relativement chères), fréquente peu les spectacles ${ }^{13}$. C'est lors des gratis offerts par le roi pour célébrer telle ou telle grande occasion nationale (annonce de la paix, naissance du dauphin) que le peuple envahit littéralement le théâtre ${ }^{14}$.

10 Dans le Paysan parvenu de Marivaux, le comte d'Orsan, que Jacob vient de sauver de la mort, lui explique qu'il se battait en duel à cause d'«une femme que je rencontrai il y a quelques jours à l'Opéra. Je la remarquai d'une loge où j'étais avec des hommes; elle me parut extrêmement jolie, aussi l'est-elle; je demandai qui elle était, on ne la connaissait pas. Sur la fin de l'Opéra je sortis de ma loge pour aller la voir sortir de la sienne, et la regarder tout à mon aise» (texte établi, avec introduction, bibliographie, chronologie, notes et glossaire par Frédéric Deloffre, édition revue avec la collaboration de Françoise Rubellin, Paris, Bordas, coll. «Classiques Garnier», 1992, p. 257). Comme on le voit, l'opéra représenté n'est qu'un prétexte au réel spectacle que constitue la jolie femme, au point que le comte $\mathrm{d}^{\prime}$ Orsan ne rappelle même pas le titre de l'œuvre vue et sort avant la fin.

11 "Je vis tout à coup braquer de toutes parts un tas de lorgnettes, qui allaient pénétrer dans chaque loge, pour découvrir quelles beautés y étaient. Les contenances, les visages, les ajustements, tout était matière à leur critique [...]. Pour m'éclairer, j'examinai scrupuleusement ces lorgneurs. Car les plus jeunes me paraissaient les plus empressés à se servir de ces lorgnettes" (suite apocryphe du Paysan parvenu, op. cit., p. 284). Ainsi, en ricochet, les spectatrices composent-elles le spectacle des lorgneurs qui constituent eux-mêmes un spectacle pour Jacob.

12 Abbé Prévost, Histoire de Manon Lescaut, Paris, Classiques Garnier, p. 49.

13 La rue elle-même avec ses processions, ses entrées solennelles, ses enterrements, offre un spectacle permanent et gratuit.

14 Les précisions numéraires données effraient lorsque l'on songe qu'habituellement une loge contenait à grand-peine huit personnes; le taux de remplissage est de quatre fois la normale! 
En juin 1744, elle persuade ses amis «d'aler voir le taudis de la comedie gratis», l'entrée étant gratuite à la Comédie-Française pour célébrer la victoire de Menin:

Tout etoit fini quand nous arrivames et il n'etoit pas quatre heures. On leur avoit donné Les Plaideurs et L'Avocat Patelin. Des onze heures tout etoit plein. On joua a deux pour s'en debarasser, mais on n'en fut pas quitte pour cela. Tout resta jusqu'a sept pour se voir les uns les autres, apparement, et pour dire: «J'y suis». La Petite me tira, aidée de trois ou quatre hommes, jusqu'au milieu du theatre pour voir ce monde imence. Je ne pus perser jusqu'au bout. J'eus le tems de compter la loge de la reine: ils etoient trente-deux dedans. Eh mon Dieu, nous y sommes huit tres incomodé les grands jours! Il y avoit a la plus belle place un savettier en bonnet de nuit [...]. Elles [des poissardes] danserent au bruit d'un tambourg dont une d'elle batoit, vrai tambour de soldat. Cela fut nouveau pour moi et je m'en amusai presque une demi-heure $(\mathrm{V}, 316)$.

Bien que l'horaire ait été considérablement avancé (généralement les pièces se jouent vers 4 ou 5 heures), le public demeure dans la salle fort tard, tout au plaisir de se regarder, de s'admirer dans ce lieu luxueux qui lui est d'habitude interdit: le peuple se constitue naturellement en spectacle ("pour se voir les uns les autres»), adoptant ainsi tout naturellement l'attitude des conditions plus privilégiées. On constate très nettement dans ce passage que Mme de Graffigny et ses amis ne viennent pas voir le spectacle (d'ailleurs ils arrivent après que la pièce est achevée!), mais les spectateurs eux-mêmes en tant qu'ils constituent un spectacle: de même qu'une parodie s'apprécie d'autant mieux que l'on est familier du texte parodié; de même seuls les habitués de la salle apprécient pleinement l'ironie carnavalesque que représente un savetier en bonnet de nuit (donc un homme, et en négligé qui plus est) dans la meilleure loge et la plus raffinée, celle de la reine. Par ailleurs, lors du gratis, tout un chacun peut non seulement s'asseoir dans le théâtre mais sur le thêâtre. D'habitude une sélection tacite interdit au peuple, confiné au parterre, d'infliger la vision de sa crasse et de sa pauvreté au reste de la salle. Mais l'une des actrices s'amuse à faire un casting et à constituer ces spectateurs inhabituels en spectacle, jouant sur la symétrie et l'opposition (qui se traduit sans doute visuellement, les charbonniers noirs de suie, et les meuniers blancs de farine) avec deux couples. Et Mme de Graffigny parle bien ici de personnages, comme si ces spectateurs anonymes par leur passage sur scène étaient devenus des types, les héros d'un spectacle:

Ah, j'alois bien oublier de te dire que samedi les comediens ont donné la comedie gratis. Il n'y avoit de regle que celle de faire entrer du monde de chaque etat jusqu'a douse decroteur. Il y avoit jusqu'a vingt personne dans chaque loge. Tout 
ce qui a voulu etre sur le theatre y etoit. La Gossin a trouvé plaisant de faire mettre deux fauteuils pour quatre personnages ${ }^{15}$ qu'elle a remarqué, d'un coté un charbonnier et une meuniere et de l'autre une charbonniere et un m[e]unier. On a joué Porsognac et Les Trois Cousine (I, 536).

\section{Le spectacle sur le théâtre}

Jusqu'en 1759 , des spectateurs sont assis sur la scène même ${ }^{16}$. Ce voisinage donne lieu à de nombreuses anecdotes célèbres, comme celle de Mlle Dumesnil frappée par un soldat. Mais, assez curieusement, on ne trouve sous la plume de Mme de Graffigny que peu d'allusions à cette présence, sans doute à la fois parce que ce public placé sous le feu de tous les regards se conduisait de façon policée en général ${ }^{17}$, et aussi parce que cette présence allait tellement de soi pour elle qu'elle omet de la signaler. Cependant, lorsqu'elle cherche à faire monter les Portraits de Devaux ${ }^{18}$, elle n'épargne aucune peine pour lui valoir le succès et explique: «Tous nos beaux esprit sont gagné et chuchetront a l'oreille sur le theatre: "Ah, la bonne piece!" J'ai aussi une cabale au partere» $(\mathrm{IV}, 470)^{19}$. On voit nettement que le succès se gagne à la fois par le bas et par le haut, et que la réaction des spectateurs sur le théâtre et dans le parterre influence celle du reste de la salle.

15 Qu'aurait pensé Musset de cet autre type de théâtre dans un fauteuil?

16 Voir Barbara G. Mittman, Spectators on the Paris Stage in the Seventeenth and Eighteenth Centuries, Ann Arbor, Michigan, UMI Research Press, 1984.

17 Pourtant, dans la suite apocryphe du Paysan parvenu, Jacob, assis sur le théâtre, explique qu'en raison du bruit qui l'entoure il ne peut entendre la pièce: «J'écoutais souvent les acteurs sans pouvoir entendre leurs paroles. Un petit-maître se levait, se tournait pour débiter en secret, à sa droite ou à sa gauche, une sornette qu'il aurait été fâché de ne pas faire passer d'oreilles en oreilles. Le ton haut avec lequel il la débitait, paraissait dire à tous ses voisins: Si je veux bien donner à mon ami une preuve de mon affection, en lui confiant mon secret, je ne vous crois pas indignes de le partager. Oui, je continue sur ce ton, vous pouvez l'entendre; mais l'apparence de mystère que j'emploie doit suffire pour ne pas me taxer d'indiscrétion" (op. cit., p. 285).

18 Mais la pièce ne sera finalement jouée que bien plus tard.

19 Elle avait précisé plus tôt qu'elle s'occupait également du parterre: «un abbé ami de l'Abbé dont je t'ai parlé, pillier de theatre, bien de l'esprit et qui sera un de mes souteneur au partere» (IV, 234); “Je ramasse aussi des partere; j'en ai deja plus d'un» (IV, 234). 


\section{Le spectacle des personnalités}

Lorsque des spectateurs de marque (auteurs, personnalités politiques) assistent à une représentation, ils sont applaudis par le public, qui marque ainsi son admiration et les remercie d'ajouter au brillant du spectacle. Voltaire est applaudi avec frénésie à la suite de la première représentation de Mérope (20 février 1743):

On a cru voir Voltaire a la loge grillée a droite; le parterre s'est tourné et crioit a tuè-tete: «L'auteur, qu'il paroisse», et les mains d'aller. Il est desendu et a eté dans un balcon a gauche ou etoit son Émilie, on se retourne et on fait un bruit a tout rompre. Encore chose non avenue: le theatre, les loges et l'emphitheatre devienent partere, se tournent et font le même bruit (IV, 155).

Les convenances hiérarchiques et la réserve qui réglent d'ordinaire le maintien des différents publics aristocratiques s'effacent dans un enthousiasme général digne du parterre. Lors de la reprise de cette même Mérope, le 3 Février 1744, l'auteur et sa compagne sont aperçus dans une loge. Sachant qu'elle va être regardée, Mme du Châtelet a manifestement pris grand soin de sa toilette, mais elle ne convainc guère Mme de Graffigny, qui la trouve puérile et ridicule:

Il y avoit tant et tant de monde que l'on chassa les violons de l'orquestre; ils jouerent deriere le theatre. Le Monstre ${ }^{20}$ y etoit et, comme de raison, vis-a-vis de moi, car cela ne peut etre autrement. Elle etoit mise et frisée comme une petite fille qui sort du couvent et que l'on pare pour la montrer. Son ami arriva deriere elle. Il fut salué du public pendant un quart d'heure; elle le tirailloit, parce qu'il se cachoit. Elle regardoit le partere au moment de lui parler. Cela etoit fort plaisant et faisoit une bonne scene (IV, 482).

Encore une fois, Mme de Graffigny croque avec vivacité la scène muette (et elle emploie bien le terme théâtral scène) que Mme du Châtelet joue avec un Voltaire plus ou moins consentant à destination de la salle.

20 Doux surnom (avec aussi la Mégère...) que Mme de Graffigny donne à Mme du Châtelet depuis le pénible épisode de Cirey où la prolixe épistolière, accusée d'avoir répandu des vers de la Pucelle, avait été quasiment chassée. Depuis, la compagne de Voltaire lui voue une haine aussi farouche qu'injuste. Pour comble de malheur, elle se retrouve fréquemment près d'elle au théâtre. "Je ne saurois mettre les pieds a un spectacle tel qu'il soit que je n'ai le Monstre pour vis-a-vis» (IV, 362), constate-t-elle avec fatalité. 
Lorsque l'épistolière se propose d'aller assister à la reprise de Mérope, elle se réjouit autant de voir les mines de la belle Émilie que la pièce même: «Lundi le partere fit les memes exclamations pour demander l'auteur que la premiere fois [lors de la reprise], et Mde du Ch. fit les meme mines de conversation avec le partere. Cela est fort bon a voir, a ce qu'on dit. J'irai samedi» $(\mathrm{V}, 79)$.

Ailleurs, Mme de Graffigny évoque la venue à la Comédie-Française du duc et de la duchesse de Chartres:

On les a claqués en entrant, en sortant. Elle est charmante pour sa vivacité, cette petite poupée, et bien jolie. Elle a salué le theatre, les loges, le parterre et le paradis en sortant. On ne lui a repondus que des mains, mais le tique-tac a duré lontems (VI, 41).

Les grands guerriers connaissent aussi même succès: "À propos, l'Abbé m'a mandé cet aprés-diner que le comte de Saxe avoit eté reçu a l'Opera comme Merope a la comedie. On l'a nommé tout haut en le priant de se montrer: il a eté claqués d'un bel air» (IV, 173). Maurice de Saxe s'était vaillamment comporté lors de la prise d'Egra et de Prague.

\section{Le spectacle du foyer ou chauffoir}

Mme de Graffigny ne mentionne que par ouï-dire ce qui s'y passe, car c'est un lieu réservé aux hommes. Mais le comportement du marquis d'Adhémar -

Il est argenté comme un porte-mouchette et se donne des ridicule partout. Une dame chez qui Nini l'a mené le trouve joli de l'esprit, mais choquant par ses manieres. Deux ou trois esprit $m$ 'ont dit qu'il faisoit le spectacles des foyer par son air composé et ses propos apreté $(\mathrm{V}, 88)-$,

qui, par ses ridicules, devient lui-même un spectacle, rappelle irrésistiblement celui de Jacob à la fin du Paysan parvenu.

\section{Le spectacle des loges grillées}

Les loges grillées, lieu d'élection des intrigues galantes, conviennent à ceux qui ne se rendent au théâtre ni pour voir ni pour être vus. Mme de Graffigny abonde en sous-entendus lorsqu'elle évoque la loge que Mme de Preysing partage avec Cahusac: «Elle lui fit griller la loge, ou elle fut seule avec la Carpe. Je ne repondrois pas de ce qui se passa» $(\mathrm{V}, 313)$. De 
facto, ce qui se passe dans ces loges est en général discret et invisible, mais il arrive que certains rendez-vous très privés tournent en scènes publiques. Ainsi le paradis se transforme-t-il en enfer lorsqu'une rivale dépitée, s'aidant de charcuterie, à la veille du Carême qui plus est, transforme un rendez-vous amoureux en viol rabelaisien. Ici, Mme de Graffigny nous livre un épisode scabreux et piquant qu'on pourrait intituler «De cochonneries en cochonailles»:

Elle me conta une histoire dont je vais te regaler ${ }^{21}$. Le dimanche gras une dame dont tout le monde connoit le nom, etant au bal de l'Opera, s'arangeoit pour un rendezvous avec un Mr de La Carte, agreable du tems, sans s'apercevoir qu'une rivale ecoutoit. Le rendez-vous etoit pour le Mardi gras au meme bal. On etoit convenu des dominos. La rivale s'ajuste en homme ave[c] tous les signes de recconoissance et ne manqua pas de conduire la donneuse de rendez-vous au paradis. La dans un coin elle fait tout ce que l'amant le plus vif auroit fait et meme par-dela, car voiant que la patiante s'impatiantoit et se plaignoit de douleurs tres vives, elle s'enfuit et laissa ou il etoit ce qui avoit le mieux trompé la dame qui, autant par surprise de voir disparoitre la cause premiere et de sentir encore la cause seconde $\mathrm{e}^{22}$ que de l'horrible cuisson, s'evanouit. D'autre masques, peut-etre occupez moins cruellement ${ }^{23}$ qu'elle, la secoururent. Elle arracha avec peine $l^{\prime}$ instrument de son suplice que l'enflure retenoit et se fit reccondu[i]re chez elle. Noté que pour celle-ci, elle est digne de cete avanture, mais que l'autre est une prude galante, modestement et a passion. Tu peux moraliser sur la haine et la vangeance des femmes a loisir. Devine ce que c'etoit: un saussisson saupoudré de poivre et des plus fortes episses! (V, 160).

À lire la correspondance de Mme de Graffigny ${ }^{24}$, on prend bien conscience qu'au théâtre le spectacle est autant et parfois plus dans la salle (qui d'ailleurs reste allumée pendant la représentation) que sur scène. L'ingénieux Jacob l'a d'ailleurs bien compris: «Me voilà donc un peu à la mode: j'assiste maintenant à la comédie, c'est-à-dire que je fais

21 On remarque que l'emploi d'un verbe culinaire constitue ici une mise en bouche.

22 Cette description pseudo-scientifique de l'acte sexuel fait songer au premier chapitre de Candide de Voltaire où la leçon de «physique expérimentale» donnée par Pangloss à la femme de chambre trouble Cunégonde qui «vit clairement la raison suffisante du docteur, les effets et les causes".

23 On apprécie l'emploi en litote de l'adverbe.

24 En revanche, la Zilia des Lettres d'une Péruvienne ne fait nulle allusion au spectacle dans le spectacle, qu'elle décrive le théâtre (lettre XVI) ou l'opéra (lettre XVII). 
nombre au spectacle ${ }^{25}{ }$. On ne s'étonnera pas que, par un juste retour des choses, Cénie, pièce de celle qui a si bien su observer le spectacle en marge du spectacle, soit elle-même un prétexte au spectacle dans la salle. Casanova s'y rend pour repérer de futures conquêtes et susciter la curiosité par son habillement:

On donnait au théâtre italien dans ce jour-là, Cénie, pièce de Mme de Grafigni. J'y suis allé de bonne heure pour avoir une bonne place à l'amphithéâtre.

Les dames toutes chargées de diamants qui entraient dans les premières loges m'intéressaient, et je me tenais attentif à les voir. J'avais un bel habit, mais ayant les manches ouvertes, et les boutons jusqu'au bas, tout le monde qui me voyait me reconnaissait pour étranger: cette mode n'existait plus à Paris ${ }^{26}$.

Quant à Mme Denis, bien qu'elle déteste la pièce et ne comprenne pas son succès, elle s'y rend pourtant ... pour être vue: «La pièce de Mme de Graffigny a le plus grand succès. Ou je suis une sotte, ou le public se trompe. Je suis dans le doute. J'y ai déjà été trois fois et je veux qu'on m'y $v{ }^{27}{ }^{27} . »$ Les mines des petits-maîtres sur la scène, les regards et les sourires des aristocrates décolletées aux premières loges, la présence de telle ou telle personnalité, les ondes et les cris du parterre constituent autant de spectacles (avec ou sans paroles) dans le spectacle; dans cette vertigineuse galerie des glaces, chacun donne la comédie autant qu'il la regarde.

\section{CHARLOTTE SIMONIN}

Université de Nantes

25 Suite apocryphe du Paysan parvenu, op. cit., p. 287.

26 Casanova, Histoire de ma vie suivie de textes inédits, édition présentée et établie par Francis Lacassin, Paris, Robert Laffont, coll. «Bouquins», 1993, vol. I, p. 570. Casanova se trompe; il ne s'agit pas du théâtre italien, mais de la Comédie-Française.

27 Lettre à Cideville, 12 juillet 1750, citée dans Henri Lagrave, op. cit., p. 187. 